\title{
A FULL DUAL MEAN SQUARE ERROR CRITERION FOR THE EQUIVALENT LINEARIZATION
}

\author{
Nguyen Dong Anh ${ }^{*}$, Nguyen Minh Triet \\ University of Engineering and Technology, Vietnam National University, 144 Xuan Thuy Str., \\ Cau Giay Dist., Vietnam \\ "Email:ndanh10000@yahoo.com
}

Received: 3 June 2015; Accepted for publication: 19 April 2016

\begin{abstract}
Among approximate methods, the method of equivalent linearization proposed by $\mathrm{N}$. Krylov and N. Bogoliubov and extended by Caughey has remained an effective tool for both deterministic and stochastic problems. The idea of the method is based on the replacement of a nonlinear oscillator by a linear one under the same excitation. The standard way of implementing this method is that the coefficients of linearization are to be found from a criterion of equivalence. When the difference between the nonlinear function and equivalent linear one is significant the replacement leads to unaccepted errors. In order to reduce the errors one may apply the dual approach. One of significant advantages of the dual conception is its consideration of two different aspects of a problem in question allowing the investigation to be more appropriate. In this paper a special case of the weighted full dual mean square error criterion is introduced and investigated in detail. Numerical results are carried out to show that this special full dual mean square error criterion can give more accurate approximate solutions for both deterministic and random nonlinear systems.
\end{abstract}

Keywords: weighted, full dual approach, equivalent linearization, extended Duffing system.

\section{INTRODUCTION}

Nonlinear oscillator models have been widely used in many areas of physics and engineering and are of significant importance in mechanical and structural problems for the comprehensive understanding and accurate prediction of motion. The study of nonlinear systems is of interest to many researchers and various methods of solution have been proposed [1 - 3]. Among approximate methods, the method of equivalent linearization proposed by N. Krylov and N. Bogoliubov [1] and extended by Caughey [4] has remained an effective tool for both deterministic and stochastic problems. The idea of the method is based on the replacement of a nonlinear oscillator by a linear one under the same excitation. The standard way of implementing this method is that the coefficients of linearization are to be found from a criterion of equivalence [4 - 7]. In 2006, Crandall's work [8] described a number of interesting episodes in the history of the linearization technique that have arisen in the past half century. The problem of equivalent replacement plays a basic role in many fields of science and technology. The 
solution of this problem allows transforming a difficult problem to one that is much easier to be investigated. When the difference between the nonlinear function and equivalent linear one is significant the replacement leads to unaccepted errors, see for example [5 - 7]. In order to reduce the errors one may apply the dual approach recently proposed and developed in [9 - 13]. One of significant advantages of the dual conception is its consideration of two different aspects of a problem in question allowing the investigation to be more appropriate. In this paper a special case of the weighted full dual mean square error criterion [9] is introduced and investigated in detail. It is shown that this special full dual mean square error criterion can give good accurate approximate solutions for both deterministic and random nonlinear systems.

\section{FULL DUAL MEAN SQUARE ERROR CRITERION}

Let first reconsider briefly the basic idea of the classical mean square error criterion. Let consider a nonlinear system described by the following equation

$$
\ddot{x}(t)+2 h \dot{x}(t)+\omega_{0}^{2} x(t)+g(\dot{x}(t), x(t))=f(t),
$$

where $h$ and $\omega_{0}$ are constants, $g(\dot{x}, x)$ is a nonlinear function of two arguments $x, \dot{x} ; f(t)$ is periodic or random excitation. The corresponding equivalent linear equation is

$$
\ddot{x}(t)+2 h \dot{x}(t)+\omega_{0}^{2} x(t)+\alpha \dot{x}(t)+\beta x(t)=f(t),
$$

where $\alpha, \beta$ are equivalent linearization coefficients. The equation error is

$$
e(x, \dot{x})=g(x, \dot{x})-b \dot{x}-k x .
$$

According to the classical mean square error criterion one requires

$$
J_{c l}=\left\langle(g(x, \dot{x})-\alpha \dot{x}-\beta x)^{2}\right\rangle \rightarrow \min _{\alpha, \beta} .
$$

The symbol $<\cdot>$ is a corresponding averaging operator. In the case of periodic functions with the period $T$ one gets

$$
<\cdot>=\frac{1}{T} \int_{0}^{T}(\cdot) d t .
$$

In the case of random functions $\langle\cdot\rangle$ is the mathematical expectation operator. The condition (4) leads to the following coefficients of equivalence linearization

$$
\alpha=\frac{\langle g(x, \dot{x}) \dot{x}\rangle}{\left\langle\dot{x}^{2}\right\rangle}, \beta=\frac{\langle g(x, \dot{x}) x\rangle}{\left\langle x^{2}\right\rangle} .
$$

It is observed that the classical mean square error criterion gives good approximate solutions for systems with small nonlinearity. Thus, several criteria have been proposed to increase the accuracy of the equivalent linearization method [5 - 8]. A new approach to problems of nonlinear vibration and structural control was recently proposed by N. D. Anh [9]. This new approach, called dual approach, has a conception of balance in research, following the essence of problem. Using the dual approach to the problem of equivalent linearization the following weighted full dual mean square criterion was introduced in [9] 


$$
\begin{aligned}
& J_{f d}=(1-p-q)<(g(x, \dot{x})-\alpha \dot{x}-\beta x)^{2}>+p<(\alpha \dot{x}+\beta x-\mu g(x, \dot{x}))^{2}>+ \\
& +q<((1-\mu) g(x, \dot{x}))^{2}>\rightarrow \min _{\alpha, \beta, \mu}
\end{aligned}
$$

where $p, q$ are constant weighting parameters. The terminology "full" is introduced here to indicate that the objective function (7) contains 3 terms. The first term in (7) presents the forward replacement from the original nonlinear function to its equivalent linearization part. The second term in (7) presents the return (dual) replacement from the equivalent linearization part to the original nonlinear function, and the last term in (7) requires that the original and return (dual) nonlinear functions should be close each to other as much as possible. It is seen that (7) leads to the classical, $(p=q=0)$, and dual mean square error criteria, $(p=1 / 2, q=0)$, investigated in [10-11], respectively. It should be noted that the classical mean square error criterion contains only one replacement and the dual mean square error criterion investigated in [10-11] contains 2 replacements. In this paper the following case $p=q=1 / 3$ is introduced and, hence, consider the following full dual mean square criterion

$$
\begin{aligned}
& J_{1 / 3}=\frac{1}{3}<(g(x)-\beta x)^{2}>+\frac{1}{3}<(\beta x-\mu g(x))^{2}>+ \\
& +\frac{1}{3}<((1-\mu) g(x))^{2}>\rightarrow \min _{\beta, \mu} .
\end{aligned}
$$

Here, for simplicity, it is supposed that the nonlinear function depends only on $x$. Taking partial derivatives of $J_{1 / 3}$ respect to $\beta, \mu$ one gets

$$
\begin{aligned}
\frac{3}{2} \frac{\partial}{\partial \beta} J_{1 / 3} & =<(g(x)-\beta x)(-x)>+<(\beta x-\mu g(x)) x)>=0, \\
\frac{3}{2} \frac{\partial}{\partial \mu} J_{1 / 3} & =<(\beta x-\mu g(x))(-g(x))>-(1-\mu)<g^{2}(x)>=0 .
\end{aligned}
$$

Solving this system yields

$$
\beta=\frac{3}{4-r^{2}} \frac{<g(x) x)>}{<x^{2}>}, \mu=\frac{2+r^{2}}{4-r^{2}},
$$

where it is denoted

$$
r^{2}=\frac{<g(x) x>^{2}}{<g^{2}(x)><x^{2}>}
$$

Thus, the corresponding equivalent linearization coefficient $\beta$ is defined by (10) where $r^{2}$ is determined by (11). Substituting (10) into (2) leads to the corresponding equivalent linear equation as follows

$$
\ddot{x}(t)+2 h \dot{x}(t)+\left(\omega_{0}^{2}+\frac{3}{4-r^{2}} \frac{<g(x) x)\rangle}{\left\langle x^{2}\right\rangle}\right) x(t)=f(t) .
$$

Hence, the approximate response of the nonlinear system (1) where the nonlinear function $\mathrm{g}$ depends only on $\mathrm{x}$ can be found from the linear equation (12). In the next section the accuracy of the full dual mean square error criterion (8) is examined for some typical nonlinear systems.

\section{NUMERICAL EXAMPLES}

Example 1. Free vibration of extended Duffing oscillator with high nonlinearity

Consider the extended Duffing system of high nonlinearity 
Table 1. Comparison of frequencies.

\begin{tabular}{|c|c|c|c|c|c|}
\hline$n$ & $\omega_{e}$ & $\omega_{c l}$ & Error $(\%)$ & $\omega_{f d}$ & Error (\%) \\
\hline 1 & $0.847 \sqrt{\gamma}$ & $0.866 \sqrt{\gamma}$ & $2 \%$ & $0.852 \sqrt{\gamma}$ & $1 \%$ \\
\hline 2 & $0.747 \sqrt{\gamma}$ & $0.791 \sqrt{\gamma}$ & $6 \%$ & $0.765 \sqrt{\gamma}$ & $2 \%$ \\
\hline 3 & $0.675 \sqrt{\gamma}$ & $0.740 \sqrt{\gamma}$ & $10 \%$ & $0.707 \sqrt{\gamma}$ & $5 \%$ \\
\hline 4 & $0.620 \sqrt{\gamma}$ & $0.702 \sqrt{\gamma}$ & $13 \%$ & $0.664 \sqrt{\gamma}$ & $7 \%$ \\
\hline 5 & $0.577 \sqrt{\gamma}$ & $0.672 \sqrt{\gamma}$ & $16 \%$ & $0.631 \sqrt{\gamma}$ & $9 \%$ \\
\hline 6 & $0.542 \sqrt{\gamma}$ & $0.647 \sqrt{\gamma}$ & $19 \%$ & $0.605 \sqrt{\gamma}$ & $12 \%$ \\
\hline 7 & $0.512 \sqrt{\gamma}$ & $0.627 \sqrt{\gamma}$ & $22 \%$ & $0.583 \sqrt{\gamma}$ & $14 \%$ \\
\hline
\end{tabular}

$$
\ddot{x}+\gamma x^{2 n+1}=0,
$$

with initial conditions $x(0)=1, \dot{x}(0)=0$, where $\gamma$ is a positive constant. For $\mathrm{n}=1$, the system (13) is known as the Duffing oscillator. The periodic vibration exists for the conservative system (13) and its exact frequency is determined by the formula [1]

$$
\omega_{e}=\frac{2 \pi}{T_{e}}, T_{e}=4 \int_{0}^{1} \frac{d x}{\left[2 \int_{x}^{1} \gamma x^{2 n+1} d x\right]^{1 / 2}} .
$$

Using (6), (12) one gets frequencies obtained by the classical (4) and full dual (8) criteria, respectively,

$$
\begin{aligned}
& \omega_{c l}=\sqrt{\frac{\gamma\left\langle x^{2 n+2}(t)\right\rangle}{\left\langle x^{2}(t)\right\rangle}=\sqrt{\frac{\gamma\left\langle\cos ^{2 n+2}\left(\omega_{c l} t\right)\right\rangle}{\left\langle\cos ^{2}\left(\omega_{c l} t\right)\right\rangle}}} \\
& \omega_{f d}=\sqrt{\frac{3}{4-r^{2}} \frac{\left\langle\cos ^{2 n+2}\left(\omega_{f d} t\right)\right\rangle}{\left\langle\cos ^{2}\left(\omega_{f d} t\right)\right\rangle}}, r^{2}=\frac{\left\langle\cos ^{2 n+2}\left(\omega_{f d} t\right)\right\rangle^{2}}{\left\langle\cos ^{4 n+2}\left(\omega_{f d} t\right)\right\rangle\left\langle\cos ^{2}\left(\omega_{f d} t\right)\right\rangle} .
\end{aligned}
$$

The comparison between the approximate frequencies (15), (16) with exact one is shown in Tab.1, in which it is observed that the accuracy of (16) is much better than the one of (15) for all values of $\mathrm{n}$. In particular, the percent error of classical solution is $22 \%$ while the percent error of full dual solution is $14 \%$ for $n=7$, respectively.

Example 2. Mean square response of Duffing oscillator subjected to random excitation

Consider the forced Duffing oscillator

$$
\ddot{x}+2 h \dot{x}+\omega_{o}^{2} x+\gamma x^{3}=\sigma \xi(t),
$$

where $h, \omega_{o}, \gamma, \sigma$ are positive constants, $\xi(t)$ is a white noise with delta correlation function

$$
\langle\xi(t)\rangle=0,\langle\xi(t) \xi(t+\tau)\rangle=\delta(\tau) .
$$


The exact mean square response to the nonlinear system (17) is found as follows [5-6]

Table 2. Comparisons of mean square responses.

\begin{tabular}{|c|c|c|c|c|c|}
\hline$\gamma$ & $\left\langle x^{2}(t)\right\rangle_{\mathrm{ex}}$ & $\left\langle x^{2}(t)\right\rangle_{\mathrm{cl}}$ & Error\% & $\left\langle x^{2}(t)\right\rangle_{\mathrm{fd}}$ & Error\% \\
\hline 0.1 & 0.818 & 0.805 & $1.5 \%$ & 0.821 & $0.4 \%$ \\
\hline 0.5 & 0.579 & 0.549 & $5.3 \%$ & 0.570 & $1.6 \%$ \\
\hline 1 & 0.468 & 0.434 & $7.2 \%$ & 0.454 & $3.0 \%$ \\
\hline 5 & 0.254 & 0.227 & $10.6 \%$ & 0.240 & $5.5 \%$ \\
\hline 10 & 0.189 & 0.167 & $11.6 \%$ & 0,176 & $6.9 \%$ \\
\hline 50 & 0.090 & 0.078 & $13.3 \%$ & 0,083 & $7.8 \%$ \\
\hline 100 & 0.065 & 0.056 & $13.8 \%$ & 0,060 & $7.7 \%$ \\
\hline
\end{tabular}

$$
\left\langle x^{2}\right\rangle_{e}=\frac{\int_{-\infty}^{\infty} x^{2} \exp \left\{-\frac{4 h}{\sigma^{2}}\left(\frac{1}{2} \omega_{o}^{2} x^{2}+\frac{1}{4} \gamma x^{4}\right)\right\} d x}{\int_{-\infty}^{\infty} \exp \left\{-\frac{4 h}{\sigma^{2}}\left(\frac{1}{2} \omega_{o}^{2} x^{2}+\frac{1}{4} \gamma x^{4}\right)\right\} d x} .
$$

The equivalent linearization equation corresponding to Duffing system (17) takes the form

$$
\ddot{x}+2 h \dot{x}+\omega_{o}^{2} x+k x=\sigma \xi(t),
$$

where $k$ is the equivalent linearization coefficient. From (20) one gets [5]

$$
\left\langle x^{2}\right\rangle=\frac{\sigma^{2}}{4 h\left(\omega_{o}^{2}+k\right)} \text {. }
$$

For the classical mean square error criterion (4),

$$
k=k_{c l}, k_{c l}=\frac{\left.<\gamma x^{4}\right\rangle}{\left.<x^{2}\right\rangle}=3 \gamma<x^{2}>.
$$

For the full dual mean square error criterion (8),

$$
\begin{gathered}
k=k_{f d}, r^{2}=\frac{\left(\gamma<x^{4}>\right)^{2}}{15 \gamma^{2}<x^{6}><x^{2}>}=\frac{\left(3 \gamma<x^{2}>^{2}\right)^{2}}{15 \gamma^{2}<x^{2}>^{3}<x^{2}>}=\frac{3}{5}, \\
k_{f d}=\frac{3}{4-r^{2}} \frac{<\gamma x^{4}>}{<x^{2}>}=\frac{45}{17} \gamma<x^{2}>.
\end{gathered}
$$

The exact and approximate mean square responses of the random Duffing system (17) are shown in Tab. 2 where $\omega_{o}=1, \sigma^{2} /(4 h)=1$ and the nonlinearity coefficient $\gamma$ varies. It is obtained that the full dual mean square responses are much close to the exact ones than the classical mean square responses for all values of the nonlinearity coefficient $\gamma$. In particular, the percent error of classical solution is $13.8 \%$ while the percent error of full dual solution is $7.7 \%$ for $\gamma=100$, respectively.

\section{CONCLUSION}

Among approximate methods, the method of equivalent linearization proposed by $\mathrm{N}$. Krylov and N. Bogoliubov and extended by Caughey has remained an effective tool for both 
deterministic and stochastic problems. When the difference between the nonlinear function and equivalent linear one is significant the equivalent linearization leads to unaccepted errors. In order to reduce the errors one may apply the dual approach. One of significant advantages of the dual conception is its consideration of two different aspects of a problem in question allowing the investigation to be more appropriate. In this paper a special case of the weighted full dual mean square error criterion is introduced and investigated in detail. The investigation leads to a new equivalent linearization coefficient which is different to the classical one. Numerical results are carried out to show that this special full dual mean square error criterion can give good accurate approximate solutions for both deterministic and random nonlinear systems, for example, the percent error can be reduced from $22 \%$ to $14 \%$ for the periodic system and from $13.8 \%$ to $7.7 \%$ for the random system, respectively. Further investigation will be followed to extend this new approach to other nonlinear problems such as single and coupled flutter instability [14].

\section{REFERENCES}

1. Bogoliubov N. N., Mitropolski Yu. A. - Asymptotic methods in the theory of nonlinear oscillations, $4^{\text {th }}$ ed., Moscow (1974) (in Russian).

2. Jordan D. W., Smith P. - Nonlinear Ordinary Differential Equations, Clarendon Press, Oxford (1987).

3. Sanders J. A., Verhulst F. - Averaging Methods in Nonlinear Dynamical Systems, $2^{\text {nd }}$ ed., Springer (2007).

4. Caughey T. K. - Equivalent linearization techniques, Journal of the Acoustical Society of America 35 (1963) 1706-17112.

5. Roberts J. B., Spanos P. D. - Random Vibration and Statistical Linearization, Wiley, New York (1990).

6. Socha L. - Linearization methods for stochastic dynamic system, Lecture Notes in Physics. Springer, Berlin (2008).

7. Elishakoff I., Andrimasy L., Dolley M. - Application and extension of the stochastic linearization by Anh and Di Paola, Acta Mech. 204 (2009) 89-98.

8. Crandall S. H. - A half-century of stochastic equivalent linearization, Struct. Control Health Monit. 13 (2006) 27-40.

9. Anh N. D. - Duality in the analysis of responses to nonlinear systems, Vietnam Journal of Mechanics 32 (2010) 263-266.

10. Anh N. D., Hieu N. N., Linh N. N. - A dual criterion of equivalent linearization method for nonlinear systems subjected to random excitation, Acta Mech. 223 (3) (2012) 645-654.

11. Anh N. D., Zakovorotny V. L., Hieu N. N., Diep D. V. - A dual criterion of stochastic linearization method for multi-degree-of-freedom systems subjected to random excitation, Acta Mech. 223 (12) (2012) 2667-2684.

12. Anh N. D., Hung L. X., Viet L. D. - Dual approach to local mean square error criterion for stochastic equicvalent linearization, Acta Mech. DOI 10.1007/s00707-012-0751-8.

13. Anh N. D. - Dual Approach to averaged values of functions: Advanced formulas, Vietnam Journal of Mechanics 34 (2012) 321-325.

14. Blevins R. D. - Flow-induced vibrations. $2^{\text {nd }}$ ed. Van Nostrand Reinhold, New York (1990). 